\title{
Reconfigurable Takagi-Sugeno Fuzzy Logic Control for a Class of Nonlinear System considering Communication Time Delays on Peripheral Elements
}

\author{
H. Benítez-Pérez, F. Cárdenas-Flores, F. García-Nocetti
}

\author{
Hector Benítez-Pérez, F. Cárdenas-Flores, Fabian García-Nocetti \\ Universidad Nacional Autónoma de México \\ Departamento de Ingeniería de Sistemas Computacionales y Automatización \\ Apdo. Postal 20-726., Admón. No. 20 \\ Del. A. Obregón, México D. F., CP. 01000, México. \\ E-mail: hector@uxdea4.iimas.unam.mx
}

\begin{abstract}
Nowadays the study of faults and their consequences becomes an issue into highly safety critical computer network systems. How to bound the effects of a fault and how to tackle them into a dynamic system is still an open field. In here an approach to tackle this problem is presented. The use of Takagi-Sugeno fuzzy logic structure is given in order to accomplish two challenges, the presence of local faults and the respective time delays within a realtime distributed system. This approach is pursued as reconfigurable strategy according to communication time delays within a real-time distributed system. This approach is pursued as reconfigurable strategy according to communication delays.
\end{abstract}

Keywords: fuzzy logic, network control.

\section{Introduction}

The emergence of smart sensor and actuator technology removes the need for centralized control with feedback loops to dumb peripheral actuators replacing it with a databus connection [1]. This gives an autonomous actuator installation [2] as well as local control, self-calibration, health monitoring and reconfiguration availabilities. Several strategies for managing time delay within control laws have been studied for different research groups. For instance [3] proposes the use of a time delay scheme integrated to a reconfigurable control strategy based upon a stochastic methodology. On the other hand, [4] proposes a reconfiguration strategy based upon a performance measure from a parameter estimation fault diagnosis procedure. Another strategy has been proposed by [5] where time delays are used as uncertainties, which modify pole placement of a robust control law. In [6] presents an interesting view of fault tolerant control approach related to time delay coupling. Reconfigurable control has been studied from the point of view of structural modification since fault appearance as presented by [7] it performs a combined modification of system structure and dynamical systems as studied by [8-10]. Present approach takes time delays due to communication as deterministic measured variables. As well as actuator fault presence by modification of $B$ matrix in order to propose a Takagi-Sugeno fuzzy control with two conditions loose of local peripheral elements and the related time delays. In here fuzzy logic control (FLC) law [11] views time delays as a result of deterministic reconfigurable communications based upon scheduling algorithm. These time delays are a structural consequence determined by the insertion of new elements within communication channels due to fault appearance. In fact, fault presence is taken into account as the lost of the related peripheral element, specifically, sensor or actuator elements. The result of fault effects over the peripheral element is the lost of the related measurement, in this case, the structure of the plant is modified in terms of the lost of specific peripheral elements. As an obvious consequence control structure is modified. 
Fuzzy logic control engulf both undesirable situations by defining the time delays during fault appearance as well as the related lost of the faulty peripheral element. The goal of this paper is to define a strategy for control reconfiguration based upon communication system reconfiguration. Some considerations need to be stated in order to define this approach. First, faults are strictly local in peripheral elements, faults are tackled by just eliminating the faulty element. In fact, faults are catastrophic and local. Time delays are bounded and restrictive to scheduling algorithms. Global stability can be reached using Takagi-Sugeno fuzzy logic approach. Fuzzy logic has been a very powerfull tool to systematic modelling an design complex nolinear systems as presented by Zadeh in [23-26].

This paper is placed as a strategy for reconfigurable systems as shown in Figure 1. In fact, this paper is focused into reconfigurable control law due to the presence of local faults and time delays as consequences. Time delays are measurable and bounded according a real-time scheduling algorithm. In this case scheduling algorithm is the well known earliest deadline first algorithm (EDF). According to Figure 1, structural reconfiguration takes place as result of EDF performance and related user request. This action provokes control law modification. How this is modified is the scope of this paper by using Takagi-Sugeno approach.

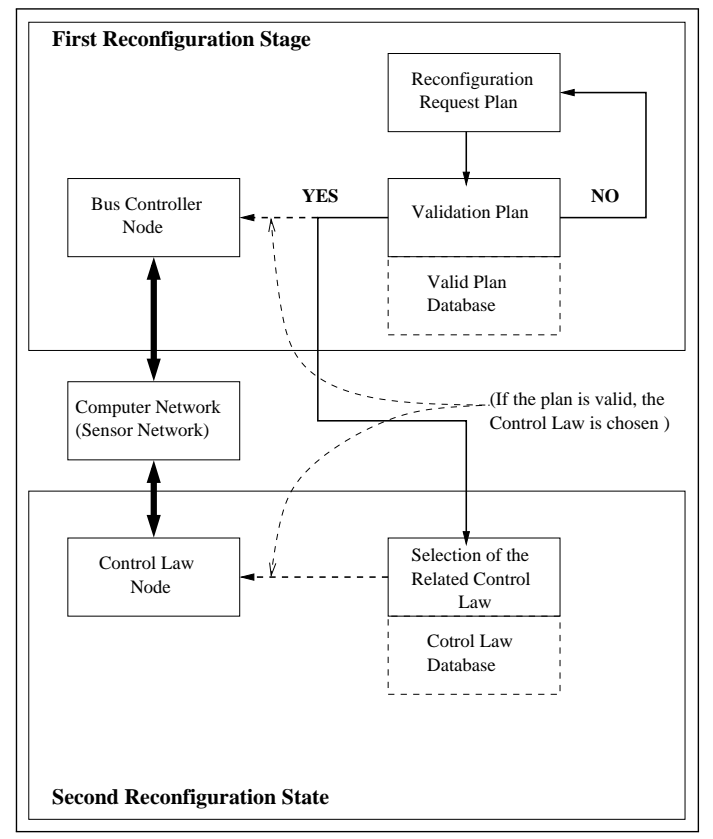

Figure 1: General structure of Reconfigurable System over a Computer Network

The map of this paper is next, first section is current introduction, second section is scheduling approach, third section is plant design, fourth section is control approach fifth section presents a case study and finally some concluding remarks are given.

\section{Scheduling aproach}

The communication network plays a key role in order to define the behaviour of the dynamic system in terms of time variance from communications and processing although it presents a nonlinear behaviour. In order to understand such a nonlinear behaviour, time delays are incorporated by the use of real-time system theory that allows time delays to be bounded even in the case of causal modifications due to external effects. Several algorithms can be pursued such as 
Rate Monotonic (RM), Deadline Monotonic or Earliest Deadline First (EDF) [15-17]. The use of last algorithm is pursued in here due to flexibility of task reorganisation during online performance. requires several characteristics from each task such as deadlines, consumption times and priorities. For instance, consider three tasks with next characteristics (Table 1 and Figure 2) first section under EDF algorithm if a task changes its deadline at $\Delta t$ it would have a higher priority than those tasks already defined (Table 2).

Table 1: Task used to exemplified EDF algorithm.

\begin{tabular}{ccccc}
\hline $\begin{array}{c}\text { Task } \\
\text { number }\end{array}$ & $\begin{array}{c}\text { Consumption } \\
\text { Time }(\mathrm{C})\end{array}$ & $\begin{array}{c}\text { Periodic } \\
\text { Time }(\mathrm{P})\end{array}$ & $\begin{array}{c}\text { Deadline } \\
(\mathrm{D})\end{array}$ & Priority \\
\hline 1 & $C_{1}$ & $P_{1}$ & $D_{1}$ & $P_{r_{2}}$ \\
2 & $C_{2}$ & $P_{2}$ & $D_{2}$ & $P_{r_{3}}$ \\
3 & $C_{3}$ & $P_{3}$ & $D_{3}$ & $P_{r_{1}}$ \\
\hline
\end{tabular}

From Figure 2 there are two scenarios, in the first task 3 has the smallest slack time $\left(t_{s 3}\right)$ therefore it has the highest priority $P_{r_{1}}$, thereafter, task 1 has next priority $\left(P_{r_{2}}\right)$ and task 2 has the lowest priority $P_{r_{3}}$ (Table 1 ).

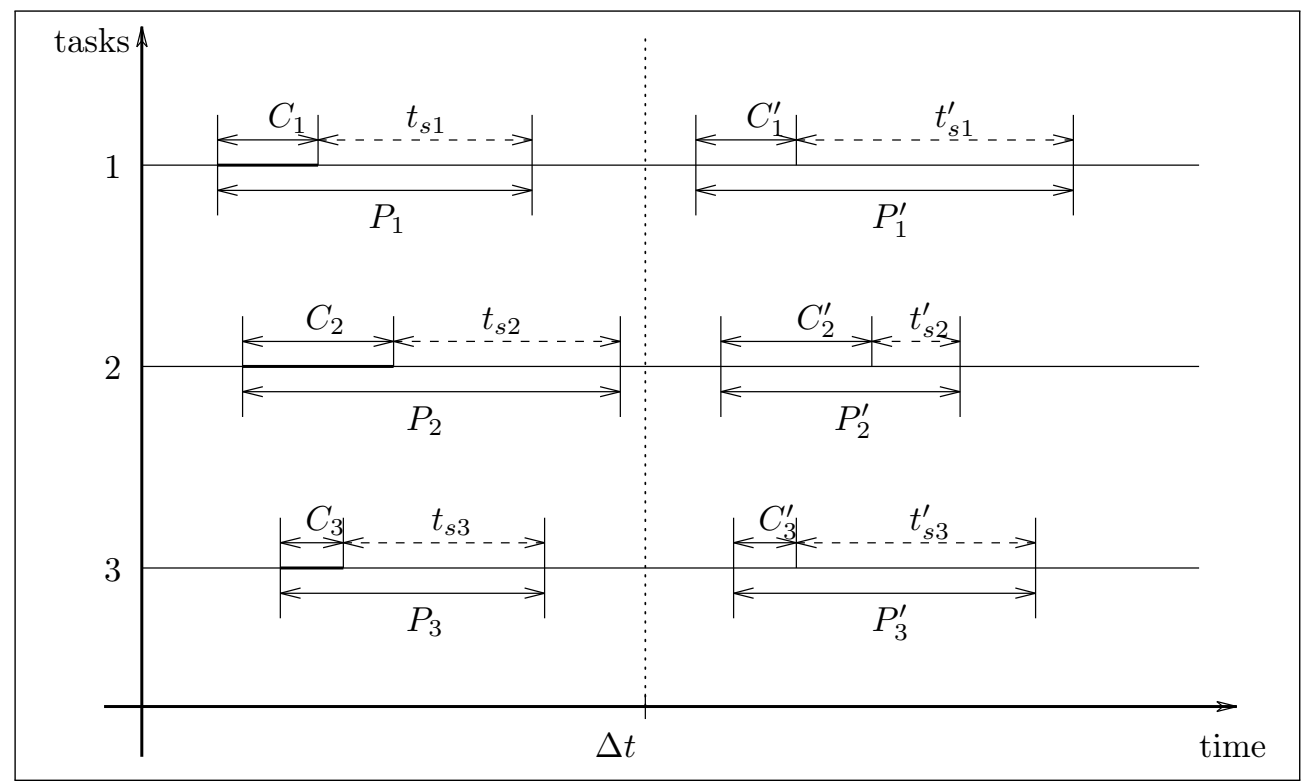

Figure 2: Time Graph Related to Table 1.

Second scenario presents a different priority conformation according to slack times modifications. Thats it for the case of deadline modification as display in Figure 2 priorities are modified as shown in Table 2 where task 2 has the smallest slack time $\left(t_{s 2}\right)$ therefore it has the highest priority $P_{r_{1}}$, task 3 has next priority and the task 1 has the lowest priority $P_{r_{3}}$. Therefore the task that is going to be executed first is the one who has the shortest deadline in comparison to the rest of alive tasks in a particular time. Since this algorithm is performed, two conditions are stated upon this approach. Control law must be executed after all sensors have performed their task and before any actuator takes an action over the plant, from these restriction system performance has two main scenarios fault present and fault free situations. These scenarios are 
Table 2: New priority order after at reorganisation.

\begin{tabular}{ccccc}
\hline $\begin{array}{c}\text { Task } \\
\text { number }\end{array}$ & $\begin{array}{c}\text { Consumption } \\
\text { Time }(\mathrm{C})\end{array}$ & $\begin{array}{c}\text { Periodic } \\
\text { Time }(\mathrm{P})\end{array}$ & $\begin{array}{c}\text { Deadline } \\
(\mathrm{D})\end{array}$ & Priority \\
\hline 1 & $C_{1}$ & $P_{1}^{\prime}$ & $D_{1}^{\prime}$ & $P_{r_{3}}$ \\
2 & $C_{2}$ & $P_{2}^{\prime}$ & $D_{2}^{\prime}$ & $P_{r_{1}}$ \\
3 & $C_{3}$ & $P_{3}^{\prime}$ & $D_{3}^{\prime}$ & $P_{r_{2}}$ \\
\hline
\end{tabular}

exposed in Figure 3, it shows a common configuration considering local time delays.

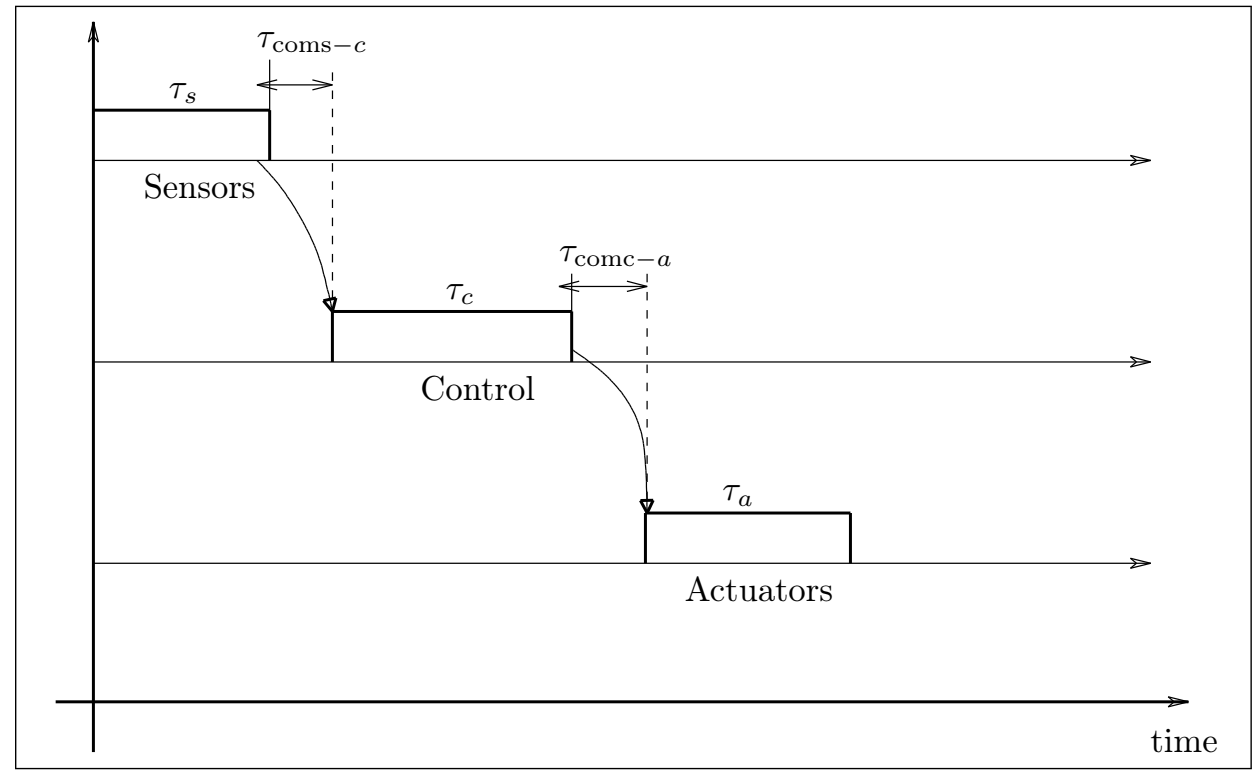

Figure 3: Related time delays from control configuration.

Related time delays are defined in terms of scheduling algorithm behaviour. Therefore time delays are stated as:

delay sensor to controller $\tau_{s c}=\tau_{s}+\tau_{\text {cons }-c}$

delay of the controller $\tau_{c}$

delay controller actuator $\tau_{c a}=\tau_{a}+\tau_{c o m c-a}$

where $\tau_{c o m s-c}$ and $\tau_{c o m c-a}$ are the source of variations due to communication variation between elements.

\section{Plant Approach}

The proposed dynamic plant is based upon the following structure:

$$
\begin{aligned}
x(k+1) & =a^{p} x(k)+B^{p} u(k) \\
y & =c^{p} x(k),
\end{aligned}
$$


where $a^{p} \in \Re^{n \times n}, c^{p} \in \Re^{n \times 1}$ and $B^{p} \in \Re^{n \times 1}$ are matrices related to the plant. $x(k), u(k)$ and $y(k)$ are the states, inputs and outputs respectively. Specially $B^{p}$ is stated as:

$$
B^{p}=\sum_{i=1}^{N} \rho_{i} B_{i} \sum_{j=1}^{M} \int_{\tau_{j}^{i}}^{\tau_{j-1}^{i}} e^{-a^{p}(t-\tau)} d \tau,
$$

where $\rho_{i} \in\{0,1\}$ and $\sum_{i=1}^{N} \rho_{i}=1$ taking into account that $N$ are the total number of possible faults and $M$ are the involved time delays from each fault. Current communication time delays are expressed as $t_{j-1}^{i}$ and $t_{j}^{i}$. Considering that $\sum_{j=1}^{M} \tau_{j}^{i} \leq T$, where $T$ is the sampling period and depends of the faults scenarios, then $B_{i}$ is integrated as:

$$
B_{i}=\left[\begin{array}{c}
b_{1} \\
b_{2} \\
0_{i} \\
\vdots
\end{array}\right] \rightarrow, i \text { fault element }
$$

where $b_{i} \rightarrow b_{n}$ are the elements conformed at the input of the plant (such as actuators) and $0_{i}$ is the lost element due to local fault where $B^{p}$ represents only one scenario following Equation 2. Therefore $B_{i}^{p}$ defined as:

$$
B_{i}^{p}=B_{i} \sum_{j=1}^{M} \int_{t_{j}^{i}}^{t_{j-1}^{i}} e^{a^{p}(t-\tau)} d \tau,
$$

considers local faults and related time delays. For simplicity purposes $B_{i}^{p}$ is used in order to depict local linear plants.

From this representation fuzzy plant is defined as follows, taking into each time delay and fault cases:

$$
r_{1} \text { IF } x_{1} \text { is } A_{1 i} \text { and } x_{2} \text { is } A_{2 i} \text { and } \ldots \text { and } x_{\ell} \text { is } A_{\ell i} \text { THEN } a_{i}^{p} x(k)+B_{i}^{p} u(k),
$$

where $x_{1} \ldots x_{\ell}$ are current state measures, $\ell$ is the number of states, $i=1, \ldots, N$ is one of the fuzzy rules, $N$ is the number of the rules which is equal to the number of possible faults and $A_{i j}$ are the related membership functions which are gaussians defined as:

$$
A_{i j}\left(x_{i}\right)=\exp \left(-\frac{\left(x_{i}-c_{i j}\right)^{2}}{\sigma_{i j}^{2}}\right)
$$

where $c_{i j}$ and $\sigma_{i j}$ are constants to be tuned. Then it can be write $h_{i}$ as follows:

$$
h_{i}=\prod_{j=1}^{\ell} A_{i j}\left(x_{j}\right)
$$

The final representation of plant as integrated system is based upon center of area defuzzification is expressed as:

$$
x^{p}(k+1)=\frac{\sum_{i=1}^{N} h_{i}\left(a_{i}^{p} x(k)+B_{i}^{p} u(k)\right)}{\sum_{i=1}^{N} h_{i}}
$$

It is important to remember Equation 2 in order to pursue system response. From this representation of a global nonlinear system, the integration of several linear systems, it is necessary to define global stability as a result of this fuzzy system. This review is given in following section considering fuzzy logic control approach. The result of this system representation allows the integration of nonlinear stages and transitions to basically a group of linear plants. 


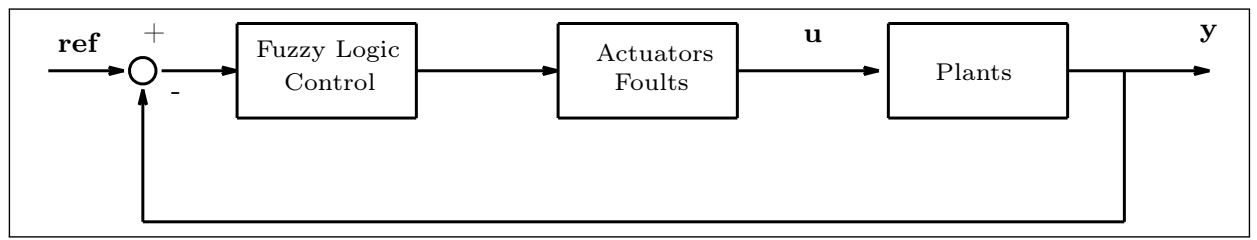

Figure 4: Plant Configuration using Fuzzy Logic Control.

\section{Control Approach}

From the representation of the plant as fuzzy system [18] it is considered to develop the control law as a group of bounded local linear control laws related to each local linear system. The structure of each fuzzy rule is:

$$
r_{1} \text { IF } x_{1} \text { is } A_{1 i}^{c} \text { and } x_{2} \text { is } A_{2 i}^{c} \text { and } \ldots \text { and } x_{\ell} \text { is } A_{\ell i}^{c} \text { THEN } u(k)=-g_{i} x(k),
$$

where $i=1, \ldots, N, \mathrm{~N}$ is the number of fuzzy rules which is the number of faults to be represented, $x_{1}, \ldots, x_{\ell}$ are current states of the plant, $A_{i j}^{c}$ are the gaussians members functions like:

$$
A_{i j}^{c}=\exp \left(-\frac{\left(x_{i}-c_{i j}^{c}\right)^{2}}{\left(\sigma_{i j}^{c}\right)^{2}}\right)
$$

where $c_{i j}^{c}$ and $\sigma_{i j}^{c}$ are constants to be tuned. Furthermore $g_{j}$ represents the related control gain. Similar to fuzzy system plant, fuzzy control representation is integrated as:

$$
w_{i}=\prod_{j=1}^{\ell} A_{i j}^{c}\left(x_{j}\right)
$$

and

$$
u(k)=\frac{\sum_{j=1}^{N} w_{i}\left(g_{i} x(k)\right)}{\sum_{i=1}^{N} w_{i}}
$$

The configuration of FLC is integrated to the already explored plant where final representation is given as closed loop system of linear feedback plant as shown in Figure 4.

$$
\begin{aligned}
x(k+1) & =\frac{\sum_{i=1, j=1}^{N} h_{i} w_{j}\left(\left(a_{i}-c_{i} g_{j} B_{i}^{p}\right) x(k)+B_{i}^{p} g_{j} \mathbf{r e f}\right)}{\sum_{i=1, j=1}^{N} h_{i} w_{j}} \\
y & =c_{i} u_{i}=c_{i} g_{j} B_{j}
\end{aligned}
$$

where ref is the reference to be followed by controller and the variables $i$ and $j$ are used due to fuzzy rules interconnections as the representation of different linear plants and theirs controllers. From this representation stability needs to be stated as the following Lyapunov function:

$$
V(x(k))=x^{T}(k) P x(k)
$$

and

$$
\Delta V(x(k))=v(x(k+1))-v(x(k))
$$


where

$$
\begin{aligned}
V(k+1)= & x(k+1)^{T} \operatorname{Px}(k+1) \\
= & \left(\frac{\sum_{i=1, j=1}^{N} h_{i} w_{j}\left(\left(a_{i}-c_{i} g_{j} B_{j}^{p}\right) x(k+1)+B_{j}^{p} g_{j} \text { ref }\right)}{\sum_{i=1, j=1}^{N} h_{i} w_{j}}\right)^{T} P \\
& \left(\frac{\sum_{i=1, j=1}^{N} h_{i} w_{j}\left(\left(a_{i} c_{i} g_{j} B_{j}^{p}\right) x(k+1)+B_{j}^{p} g_{j} \mathbf{r e f}\right)}{\sum_{i=1, j=1}^{N} h_{i} w_{j}}\right) .
\end{aligned}
$$

Therefore

$$
\begin{aligned}
\Delta V(x(k))= & \left(\frac{\sum_{i=1, j=1}^{N} h_{i} w_{j}\left(\left(a_{i}-c_{i} g_{j} B_{j}^{p}\right) x(k+1)+B_{j}^{p} g_{j} \mathbf{r e f}\right)}{\sum_{i=1, j=1}^{N} h_{i} w_{j}}\right)^{T} P \\
& \left(\frac{\sum_{i=1, j=1}^{N} h_{i} w_{j}\left(\left(a_{i} c_{i} g_{j} B_{j}^{p}\right) x(k+1)+B_{j}^{p} g_{j} \mathbf{r e f}\right)}{\sum_{i=1, j=1}^{N} h_{i} w_{j}}\right)-X^{T} P X,
\end{aligned}
$$

and

$$
\Delta V(x(k))=\frac{\left(h_{i=1, j=1}^{N} w_{j}\right)^{2} x^{T}\left[\begin{array}{c}
-\left(a_{i}^{T} P c_{i} g_{j} B_{j}^{P}\right)+a_{i}^{T} P\left(B_{j}^{P} g_{i} \text { ref }\right)- \\
\left(c_{i} g_{j} B_{j}^{P}\right)^{T} P a_{i}- \\
\left(c_{i} g_{j} B_{j}^{P}\right) P\left(B_{j}^{P} g_{j} \text { ref }\right)+ \\
\left(g_{i} B_{j}^{P} \text { ref }\right)^{T} P a_{i}- \\
\left(g_{j} B_{j}^{P} \text { ref }\right)^{T} P\left(c_{i} g_{j} B_{j}^{P}\right)+ \\
a_{i}^{T} P a_{i}+\left(c_{i} g_{j} B_{j}^{P}\right) P\left(c_{i} g_{j} B_{j}^{P}\right)+ \\
B_{j}^{P} g_{j} \mathbf{r e f} P\left(g_{j} B_{j}^{P} \text { ref }\right)
\end{array}\right] x-x P x}{\sum_{i=1, j=1}^{N}\left(h_{i} w_{j}\right)^{2}},
$$

then by considering ref $=0$

$$
\Delta V(x(k))=\frac{\sum_{i=1, j=1}^{N}\left(\left(h_{i} w_{j}\right)^{2} x^{T}\left[\begin{array}{c}
-\left(a_{i}^{T} P c_{i} g_{j} B_{j}^{P}\right)-\left(c_{i} g_{j} B_{j}^{p}\right)^{T} a_{i} \\
+a_{i}^{T} P a_{i}+\left(c_{i} g_{j} B_{j}^{P}\right) P\left(c_{i} g_{j} B_{j}^{P}\right)
\end{array}\right] x-x P x\right)}{\sum_{i=1, j=1}^{N}\left(h_{i} w_{j}\right)^{2}} .
$$

It is important to remember that:

$$
\Delta V(x(k)) \leq 0,
$$

and

$$
\left[\begin{array}{c}
-\left(a_{i}^{T} P c_{i} g_{j} B_{j}^{P}\right)-\left(c_{i} g_{j} B_{j}^{p}\right)^{T} a_{i} \\
+a_{i}^{T} P a_{i}+\left(c_{i} g_{j} B_{j}^{P}\right) P\left(c_{i} g_{j} B_{j}^{P}\right)
\end{array}\right]<0,
$$

where $P>0,\|\bullet\|$ is the Euclidean norm and it is possible to define:

$$
g_{j}>\left\|B_{j}^{P}\right\| .
$$

This condition has to be given for every single time delay and local fault appearance. Furthermore the stability and the convergence of states should be assured by the adequate selection of matrices $g_{i}$ (Equation 8) and the related parameters from both fuzzy systems. In this case recommendable procedure to follow is multi-objective optimisation in order to define those suitable values. 


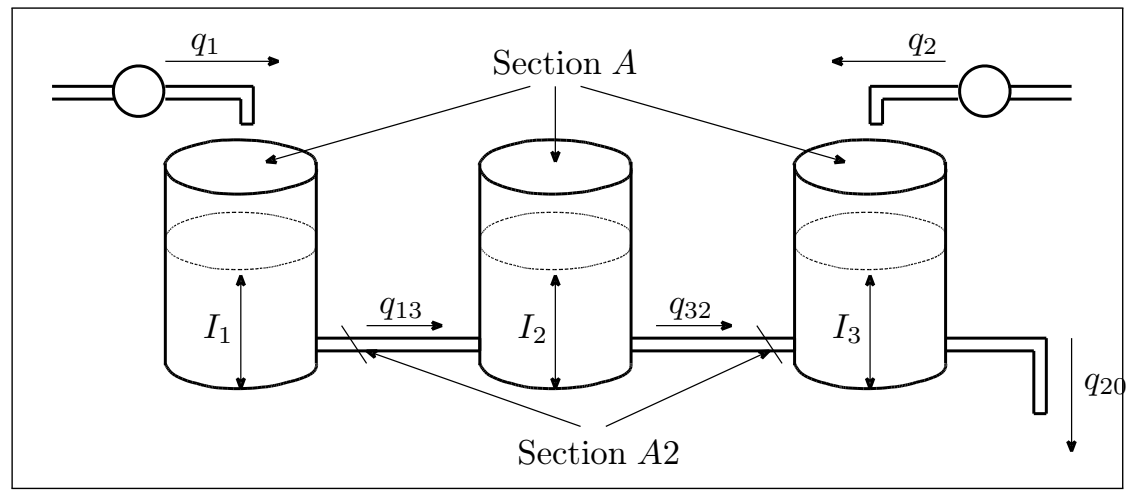

Figure 5: Three tanks representation.

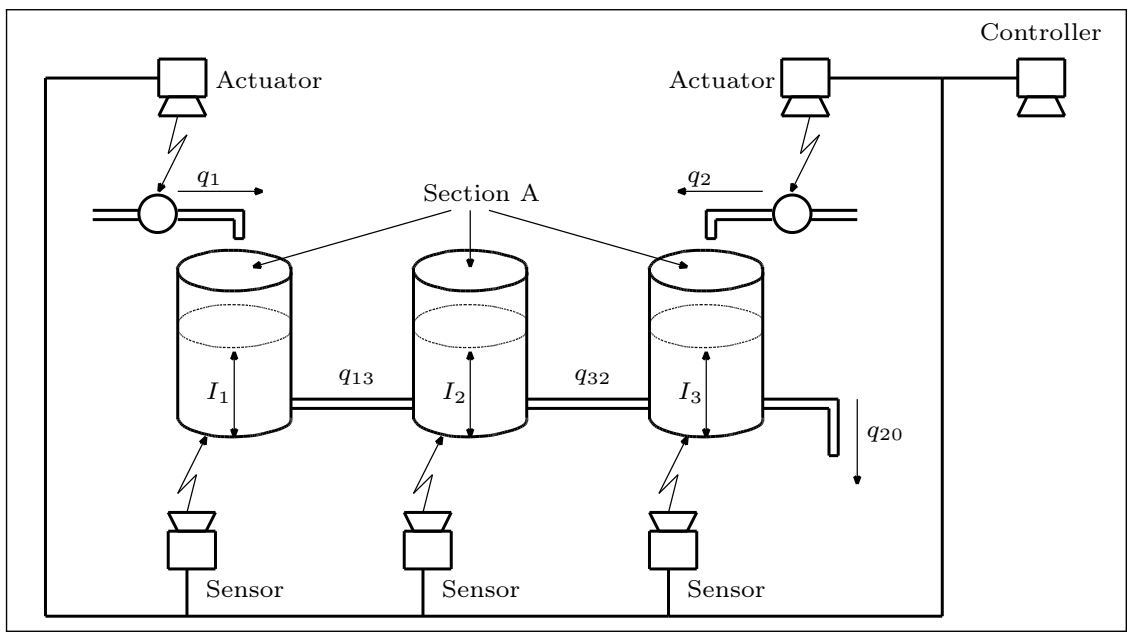

Figure 6: Three Tanks Representation based upon Computer Network.

\section{Case of Study}

Case study is based upon three tanks approach following Figure 5. This is composed by three tanks with identical cross section $A$. The tanks are coupled by two pipes with cross section of $A 2$. Two pumps are driven by DC motors who supply two inflows $q_{1}$ and $q_{2}$. Liquid level in each tank is measured and reported as $I_{1}, I_{2}$ and $I_{3}$. The computer network integration is shown in Figure 6. This system has a sampling period of $80 \mathrm{~ms}$ and nominal communication time delay of 20 ms. Common representation of this system is stated in Eq. 22 as state space representation [19]. This representation takes into account the inherent non-linearities of the model represented as:

$$
x(k+1)=\left[\begin{array}{c}
-\frac{q_{l 3}}{A} \\
\frac{q_{32} x-q_{20} x}{A} \\
\frac{q_{l 3} x-q_{32} x}{A}
\end{array}\right]+\left[\begin{array}{cc}
\frac{1}{A} & 0 \\
0 & \frac{1}{A} \\
0 & 0
\end{array}\right] U,
$$

where $x=\left[\begin{array}{lll}l_{1} & l_{2} & l_{3}\end{array}\right]^{T}, y=\left[\begin{array}{ll}l_{1} & l_{2}\end{array}\right]^{T}$ and $u=\left[\begin{array}{ll}q_{1} & q_{2}\end{array}\right]^{T}$ and

$$
\begin{aligned}
q_{i j} & =\mu_{i j} A * \operatorname{signum}\left(l_{i}-l_{j}\right) \sqrt{2 g\left|l_{i}-l_{j}\right|} \\
q_{20} & =\mu_{20} A \sqrt{2 g l_{2}} .
\end{aligned}
$$


Here $\mu_{i j}$ are the outflow coefficients. In terms of fuzzy representation, three rules are given due the possible number of faults which are two due to just two actuators are available.

$$
\begin{aligned}
& r_{i} \text { IF } x_{1} \text { is } A_{11}^{C} \text { and } x_{2} \text { is } X_{21}^{c} \text { and } x_{3} \text { is } A_{31}^{c} \text { THEN } a_{1}^{p} x(k)+B_{1}^{p} u(k), \\
& r_{2} \text { IF } x_{1} \text { is } A_{12}^{c} \text { and } x_{2} \text { is } A_{22}^{c} \text { and } x_{3} \text { is } A_{32}^{c} \text { THEN } a_{2}^{p} x(k)+B_{2}^{p} u(k), \\
& r_{3} \text { IF } x_{1} \text { is } A_{13}^{c} \text { and } x_{2} \text { is } A_{23}^{c} \text { and } x_{3} \text { is } A_{33}^{c} \text { THEN } a_{3}^{p} x(k)+B_{3}^{p} u(k),
\end{aligned}
$$

In this case $B_{1}^{p}, B_{2}^{p}$ and $B_{3}^{p}$ are defined as:

$$
\begin{aligned}
& B_{1}^{p}=B_{1}\left(\int_{0.0}^{0.03} e^{-a_{1}^{p}(t-\tau)} d \tau+\int_{0.03}^{0.08} e^{-a_{1}^{p}(t-\tau)} d \tau\right), \\
& B_{2}^{p}=B_{2}\left(\int_{0.0}^{0.04} e^{-a_{2}^{p}(t-\tau)} d \tau+\int_{0.04}^{0.08} e^{-a_{2}^{p}(t-\tau)} d \tau\right), \\
& B_{3}^{p}=B_{3}\left(\int_{0.0}^{0.05} e^{-a_{3}^{p}(t-\tau)} d \tau+\int_{0.05}^{0.08} e^{-a_{3}^{p}(t-\tau)} d \tau\right) .
\end{aligned}
$$

These three plant representations are modified just as $B$ matrix where the reconfiguration is performed. In here two integrals are presented due to important time delays of related faults. For instance, $B_{2}^{p}$ has a time delay of 0.04 and $B_{3}^{p}$ has a time delay of 0.05 seconds. In the same way control laws are represented, where three control laws are presented.

$$
\begin{aligned}
& r_{i} \text { IF } x_{1} \text { is } A_{11}^{C} \text { and } x_{2} \text { is } X_{21}^{c} \text { and } x_{3} \text { is } A_{31}^{c} \text { THEN } u(k)=-g_{1} x(k), \\
& r_{2} \text { IF } x_{1} \text { is } A_{12}^{c} \text { and } x_{2} \text { is } A_{22}^{c} \text { and } x_{3} \text { is } A_{32}^{c} \text { THEN } u(k)=-g_{2} x(k), \\
& r_{3} \text { IF } x_{1} \text { is } A_{13}^{c} \text { and } x_{2} \text { is } A_{23}^{c} \text { and } x_{3} \text { is } A_{33}^{c} \text { THEN } u(k)=-g_{3} x(k) .
\end{aligned}
$$

Based upon this equation, final closed loop equation is:

$$
x(k+1)=\frac{\sum_{i=1, j=1}^{3} h_{i} w_{j}\left(\left(a_{i}-c_{i} g_{j} B_{j}^{k}\right) x(k)+B_{j}^{k} g_{j} \mathrm{ref}\right)}{\sum_{i=1, j=1}^{3} h_{i} w_{j}} .
$$

Since this representation is given and based upon stability proposal in last section, optimisation toolbox from MATLAB [20] is used to define current values of $g$ matrices.

\section{Implementing Approach}

Following the review of case study, this is implemented over a computer network simulation $[8,21,22]$ based upon True-Time strategy. This simulation consists of a CSMA/CA CAN network integrated over ten nodes, where the typical time diagram is presented in Figure 7.

Inconsistencies appear during communication and consumption times as well as the jitter play an important role.

Table 3 shows the consumptions, periods and variations of the involved nodes where sensors and actuators are organised according to EDF algorithm. Moreover, Table 4 shows the related time delays according to Figure 6. Based upon these conditions, scheduling modifications are performed. This scheduling modification affects performance response of peripheral elements where $B_{i}^{p}$ (Equation 3) and $c_{i j}$ (Equation 5) matrices are altered as where time delays are defined as follows (Table 5): 


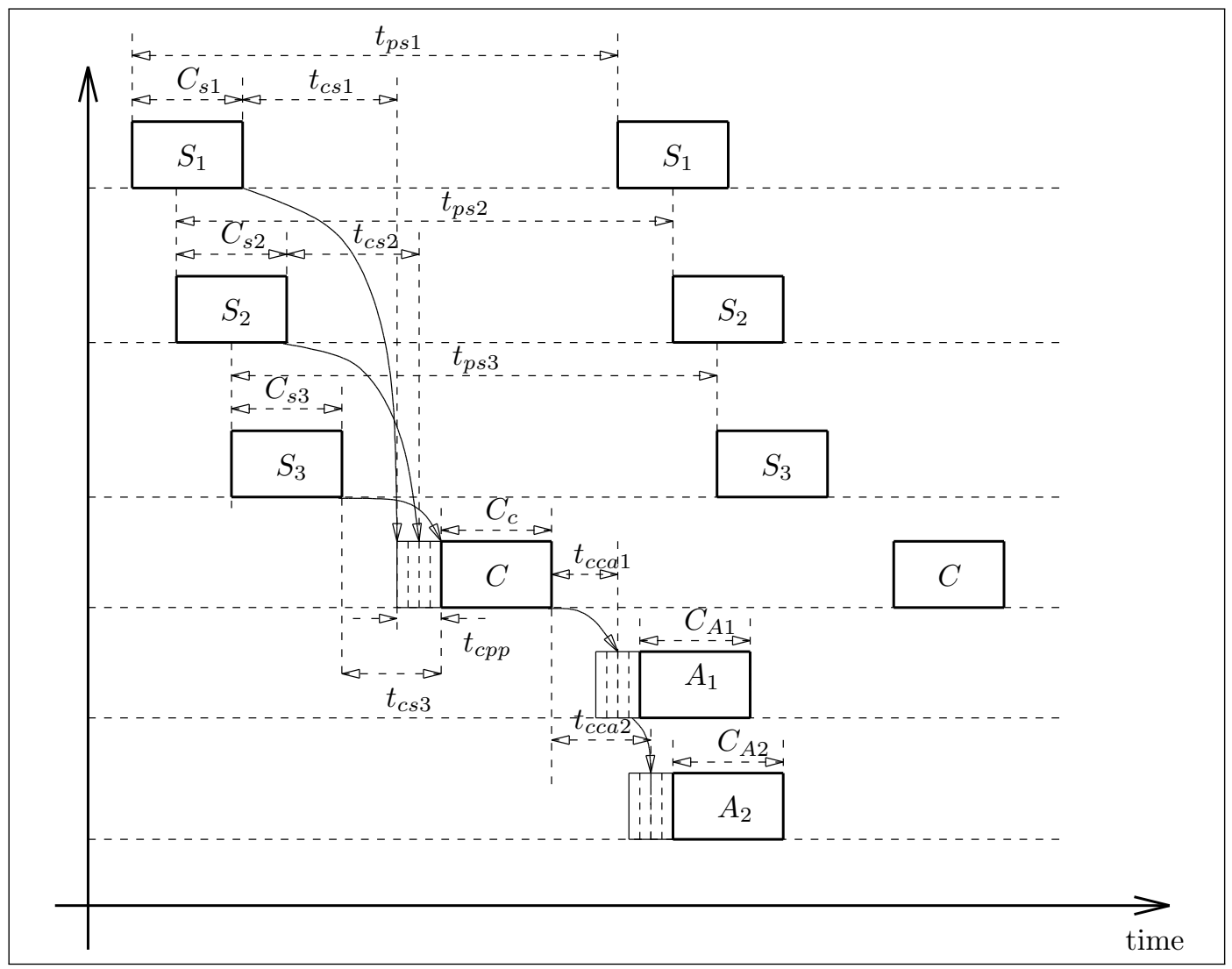

Figure 7: Time Diagram Representation based upon Computer Network.

Table 3: Consumption times and periods from tasks. (in seconds)

\begin{tabular}{ccccc}
\hline Component & $\begin{array}{c}\text { Consumption } \\
\text { time }\end{array}$ & $\begin{array}{c}\text { Variation } \\
\%\end{array}$ & $\begin{array}{c}\text { Time } \\
\text { deadlines }\end{array}$ & $\begin{array}{c}\text { Variation } \\
\%\end{array}$ \\
\hline$S_{1}$ & $C_{s 1}=0.03$ & $6-8$ & 0.08 & $2-3$ \\
$S_{2}$ & $C_{s 2}=0.03$ & $6-8$ & 0.08 & $2-3$ \\
$S_{3}$ & $C_{s 3}=0.03$ & $6-8$ & 0.08 & $2-3$ \\
$C$ & $C_{c}=0.05$ & $7-9$ & 0.08 & $2-3$ \\
$A_{1}$ & $C_{A 1}=0.045$ & $5-7$ & 0.08 & $2-3$ \\
$A_{2}$ & $C_{A 2}=0.045$ & $5-7$ & 0.08 & $2-3$ \\
\hline
\end{tabular}


Table 4: Communication Times needed (in seconds)

\begin{tabular}{cc}
\hline $\begin{array}{c}\text { Communication } \\
\text { time }\end{array}$ & $\begin{array}{c}\text { Spent } \\
\text { time }\end{array}$ \\
\hline$t_{c s 1}$ & 0.02 \\
$t_{c s 2}$ & 0.02 \\
$t_{c s 3}$ & 0.02 \\
$t_{c c a 1}$ & 0.02 \\
$t_{c c a 2}$ & 0.02 \\
jitter & 0.015 \\
\hline
\end{tabular}

$$
\begin{aligned}
& \tau_{1}^{1}=0, \tau_{2}^{1}=t_{c c a 1}+\text { jitter }+ \text { variation, and } \tau_{3}^{1}=0.08 \\
& \tau_{1}^{2}=0, \tau_{2}^{2}=t_{c c a 2}+\text { jitter }+ \text { variation, and } \tau_{3}^{2}=0.08
\end{aligned}
$$

Therefore control reconfiguration becomes necessary in order to keep certain response level. For this case study three different control laws are proposed as shown in Table 6. The type of membership functions are gaussians normally distributed over the rank of each state as shown in Figure 8. In the case that any state is gone further its effected are reduced through the related control law.

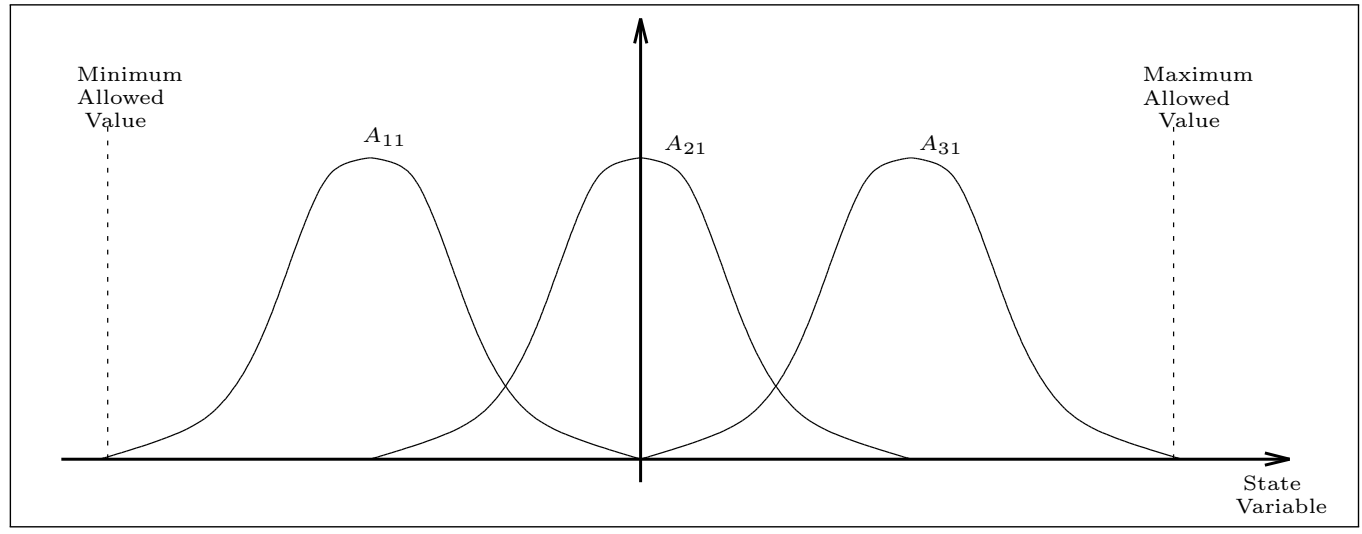

Figure 8: State Variable.

Table 5: Fuzzy logic rules considering the related plant.

\begin{tabular}{cc}
\hline Rule & Linearised Plant \\
\hline if $x_{1}$ is $A_{11}$ and $x_{2}$ is $A_{12}$ and $x_{3}$ and $A_{13}$ & $x(k+1)=a_{1}^{p} x(k)+B_{1}^{p} u$ \\
if $x_{1}$ is $A_{21}$ and $x_{2}$ is $A_{22}$ and $x_{3}$ and $A_{23}$ & $x(k+1)=a_{2}^{p} x(k)+B_{2}^{p} u$ \\
if $x_{1}$ is $A_{31}$ and $x_{2}$ is $A_{32}$ and $x_{3}$ and $A_{33}$ & $x(k+1)=a_{3}^{p} x(k)+B_{3}^{p} u$
\end{tabular}


Table 6: Fuzzy logic rules considering the related control law.

\begin{tabular}{lc}
\hline Rule & Control Law \\
\hline if $x_{1}$ is $A_{11}$ and $x_{2}$ is $A_{12}$ and $x_{3}$ and $A_{13}$ & $U=g_{1} X$ \\
if $x_{1}$ is $A_{21}$ and $x_{2}$ is $A_{22}$ and $x_{3}$ and $A_{23}$ & $U=g_{2} X$ \\
if $x_{1}$ is $A_{31}$ and $x_{2}$ is $A_{32}$ and $x_{3}$ and $A_{33}$ & $U=g_{3} X$ \\
\hline
\end{tabular}

\section{Results}

Following the response of the case study, some interesting results are given related to three different scenarios named reconfiguration (stages) 1, 2 and 3 (Figure 9a), where each stage is $20 \mathrm{~ms}$ long. And for each of the those cases three different responses like: dynamic response, consumption and communication times are given. The response shown, corresponds to three tanks performance during three different dynamic conditions with respect to communication time behaviour. The local response presented in the third scenario is performed due to modification of the response and dynamic conditions with respect to communication time delays. Figure 10) presents communication system response of each element involved on the system. In this case, periodic activation per task and node is presented. Where free time needs to be reorder to be more efficient. Figure 10a) presents periodic time modification when reconfiguration was necessary and second graphic presents time used to communicate elements through the network. For instance, at first 20 seconds every period of activated task are relatively similar. From 20-40 seconds those modified periodic tasks change the relatively. Similar situation is presented from 40-60 seconds. The reader may realize that some tasks reduce their periodic consumption times while others tends to spend more time.

\section{Concluding Remarks}

As has been shown in this work, fuzzy logic control based upon Takagi-Sugeno structure allows the possibility of control reconfiguration as long as linear models based representation of the plant are available. Despite of local faults and bounded time delays appearance, several conditions should be fulfil in order to be able to follow this proposal, for instance, plant should be observable and controllable during the whole nonlinear behaviour as well as the states should be present during undesirable situations. Several approaches can be pursued such as robustness study during variations from one to another scenarios as well as further work is needed when not every state is available during fault conditions which is a realistic condition. In this case observer design under fault scenario need to be defined looking for smooth transitions between fault and fault free status. Stability conditions are reached as long as system design is pursued by Equations 15-19, where fuzzy logic variables and local control laws are established. Following future work, a non-desirable drawback is related to a peripheral element fault without any propagation to the rest of the system. This can be possible by just considering some specific scenarios during fault propagation from local to general catastrophic faults.

\section{Acknowledgements}

The authors would like to thank the financial support of DISCA-IIMAS-UNAM, and UNAMPAPIIT (IN106100, IN103310 and IN101307), ICyTDF PICCO10-53 México in connection with this work. 
Reconfigurable Takagi-Sugeno Fuzzy Logic Control for a Class of Nonlinear System considering

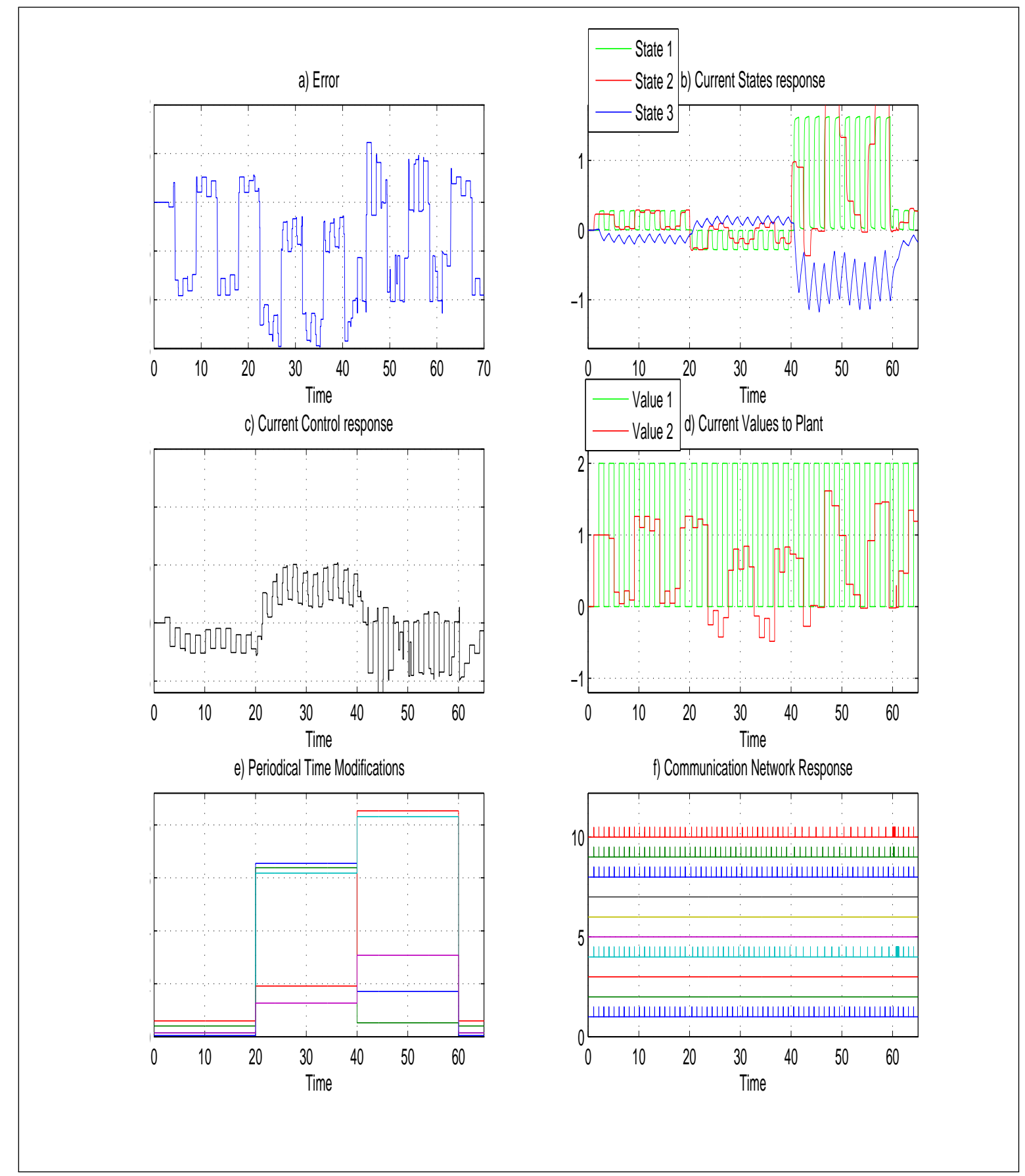

Figure 9: Dynamic Response of Case Study Following Three Scenarios. 


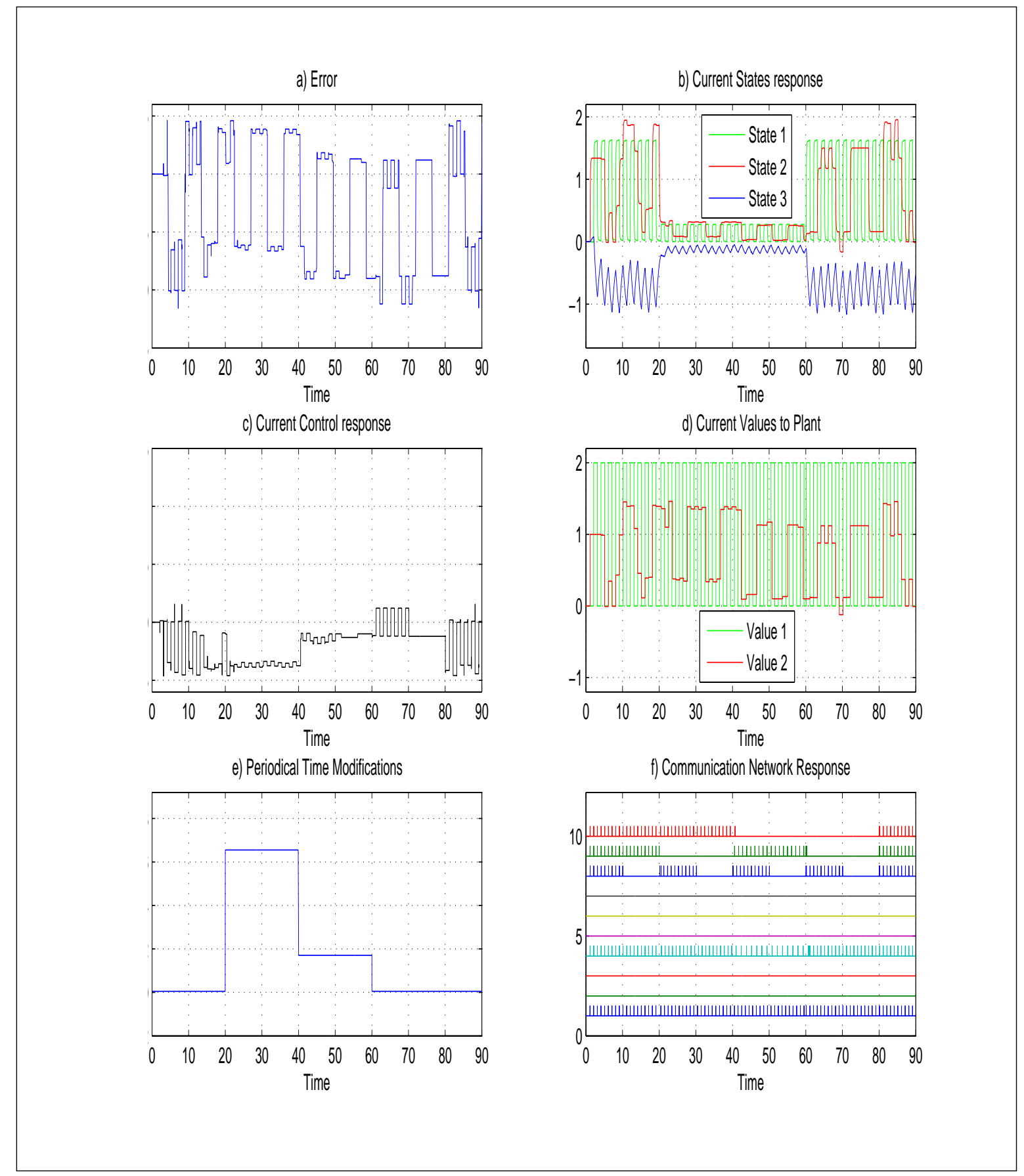

Figure 10: Dynamic Response of Case Study Following Three Scenarios considering periodic communication. 


\section{Bibliography}

[1] Benítez-Pérez, H., Thompson, H. A. and Fleming, P. J., Simulation of Distributed Fault Tolerant Heterogeneous Architectures for Real-Time Control, 5 th IFAC Workshop on Algorithms and Architectures for Real-Time Control AARTC98, pp. 89-94, Cancún, México, 1998.

[2] Masten, M. K., Electronics: The intelligence in Intelligent Control IFAC Symposium on Intelligent Components and Instrument for Control Applications, Annecy, France, pp. 1-11, 1997.

[3] Nilsson, J., Real-Time Control with Delays, PhD. Thesis, Department of Automatic Control, Lund Institute of Technology, Sweden, 1998.

[4] Wu N., Reliability of Reconfigurable Control Systems: A Fuzzy Set Theoretic Perspective, Proceedings of the 36 th Conference on Decision 83 Control, IEEE, TP15 5:10, pp. 33523356, San-Diego, USA, 1997.

[5] Jiang J., and Zhao Q.,Reconfigurable Control Based on Imprecise Fault Identification, Proocedings of the American Control Conference, IEEE, pp. 114-118, San Diego, June, 1999.

[6] Izadi-Zamanabadi R. and Blanke M.,A Ship Propulsion System as a Benchmark for FaultTolerant Control, Control Engineering Practice, Vol. 7, pp. 227-239, 1999.

[7] Blanke, M., Kinnaert M., Lunze J., and Staroswiecki M.,Diagnosis and Fault Tolerant Control, Springer, 2003.

[8] Benítez-Pérez, H., and García-Nocetti, F., Reconfigurable Distributed Control, Springer Verlag, 2005.

[9] Benítez-Pérez, H., and García-Nocetti, F., Reconfigurable Distributed Control based upon Smart Peripheral Elements, European Control Conference; ECC01, Porto, Portugal, pp. 35343539,2001

[10] Benítez-Pérez, H., Smart Distributed Systems, PhD. Thesis AC \& SE Dept. University of Sheffield, UK, 1999.

[11] Driankov, D., Hellendoorn, H., Reinfrank, M., An Introduction to Fuzzy Logic Control, Springer- Verlag, 1994.

[12] Benítez-Pérez, H., Cárdenas-Flores F., García-Nocetti, F., An Implementation of Reconfigurable Network Control based upon Automata Proposal for three Conveyor Belt Case of Study, International Journal of Computers, Communications \& Control, vol. II(2007), No. 4, pp 314-327, 2007.

[13] Benítez-Pérez, H., Real-time distributed control: A fuzzy an model predictive control approach for a nonlinear problem, Nonlinear Analysis Hybrid Systems, 2 (2008), pp. 474-490, 2008.

[14] Quiñones-Reyes P., Benítez-Pérez, H., Cárdenas-Flores F., García-Nocetti, F. An approximation for reconfigurable fuzzy Takagi-Sugeno networked control, IEEE Potentials, vol. 27, No. 6, pp. 38-44, 2008.

[15] Liu, J.,Real-Time Systems, Prentice Hall, 2000.

[16] Krishna C. M. and Shin K. G., Real-Time Systems, Mc. Graw Hill, New York, USA, 1997. 
[17] Lian F. Moyne J. and Tilbury D., Network Design Consideration for Distributed Control Systems, IEEE Transactions on Control Systems Technology, Vol. 10, No. 2, pp. 297-307, March 2002.

[18] Yi, Z., and Heng, P.; d'ż"Stability of Fuzzy Control Systems with Bounded Uncertain Delaysd'ż"; IEEE Transactions on Fuzzy Systems, vol. 10, No.1, pp. 92-97, 2002.

[19] Dardinier-Maron V., Hamelin F., and Noure H.; A Fault-Tolerant Control Design Against Major Actuator Failures: Application to a Three-Tank Systemd'ż"; IEEE 38th Conference on Decision and Control, pp: 3569-3574, 1999.

[20] Mathworks; System Identification Toolbox Users Guide; MATLAB, 1998.

[21] Cervin, A., Henriksson, D., Lincoln, B., Eker, J., and Arzd’ż”n, K.; d’ż"How Does Control Timing Affect Performance?d’ż"; IEEE Control Systems Magazine, Vol. 23, pp. 16-30, 2003

[22] Benítez-Pérez, H., and García-Nocetti, F.,Reconfigurable Takagi-Sugeno Fuzzy Logic Control for a Class of Nonlinear System considering Communication Time Delays on Peripheral Elements, Submitted to American Control Conference 2006.

[23] Zadeh L. A., A theory of commonsense knowledge, Aspects of Vagueness, H.J. Skala, S. Termini and E. Trillas (eds.), 257-296. Dordrecht: Reidel, 1984.

[24] Zadeh L. A., Fuzzy probabilities, Information Processing and Management 19, 148-153, 1984.

[25] Zadeh L. A., The Role of Fuzzy Logic in Modeling, Identification and Control, Modeling Identification and Control, 15(3):191-203, 1994.

[26] Zadeh L. A., Fuzzy Logic and the Calculus of Fuzzy If-Then Rules, Proceedings of SYNAPSE '91, Tokyo, Japan, 1991. 